\title{
Carnets
}

Revue électronique d'études françaises de l'APEF

Deuxième série - 4 | 2015

Regards sur Camus

\section{Relire Camus : une ethnocritique de la peste}

\section{Benkhodja Ammar}

\section{(2) OpenEdition}

\section{Journals}

Édition électronique

URL : http://journals.openedition.org/carnets/1597

DOI : 10.4000/carnets. 1597

ISSN : 1646-7698

Éditeur

APEF

\section{Référence électronique}

Benkhodja Ammar, «Relire Camus : une ethnocritique de la peste», Carnets [En ligne], Deuxième série - 4 | 2015, mis en ligne le 30 mai 2015, consulté le 10 décembre 2020. URL : http:// journals.openedition.org/carnets/1597 ; DOI : https://doi.org/10.4000/carnets.1597

Ce document a été généré automatiquement le 10 décembre 2020.

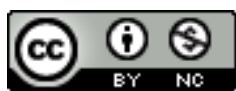

Carnets est mis à disposition selon les termes de la licence Creative Commons - Atribution - Pas d'utilisation commerciale 4.0 International. 


\title{
Relire Camus : une ethnocritique de la peste
}

\author{
Benkhodja Ammar
}

1 S'agissant du projet scriptural de La Peste, roman qui demandera à l'auteur une attention particulière ${ }^{1}$, Albert Camus, comme tout écrivain soucieux de son esthétique, s'interroge lui-même, dans ses Carnets, sur le projet d'écriture de son roman. Il décline, dans les avant-textes que constituent ses Carnets ${ }^{2}$, une hésitation double; hésitation sur le titre de son futur livre, et sur le genre littéraire qui prendra en charge sa forme: «Peste ou aventure. Roman.» (Camus, 1962 : 166), «Roman. Ne pas mettre 'La Peste' dans le livre. Mais quelque chose comme 'les prisonniers'" (Camus, 1964:34), «La Peste. Pittoresque et descriptives - petits morceaux documentaires et une dissertation sur les fléaux (...) il faut décidemment que ce soit une relation, une chronique, mais de problèmes cela pose " (Camus, 1964 : 55). Il multiplie les moyens pour faire ressortir « le sens social de la peste » (Camus, 1964: 57): "les événements de la chronique» (Camus, 1964: 57), prêches, documents médicaux... Bref, tout un arsenal documentaire à la mode zolienne qui fera de ce roman une œuvre concentrée.

2 C'est justement cette « concentration » culturelle du roman de La Peste que nous avons voulu interroger ici, en faisant l'hypothèse qu'une polyphonie des voix/voies culturelles traverse ce roman ${ }^{3}$.

3 A cet effet, nous nous intéresserons à la conception que donne le roman du fléau dont il porte le nom. Nous pensons que différents degrés de croyances y sont cristallisés, faisant s'entrecroiser différents mondes et modes de représentations symboliques plus ou moins hétérogènes, permettant de penser, et de repenser, la structure composite de la société et de la culture de l'Algérie française, et le rapport problématique qui lie l'Algérie à la France, ainsi que celui qui lie l'auteur à ces/ses deux parties.

4 Nous ne comptons pas, bien évidemment faire table rase de toutes les lectures, aussi riches les unes que les autres, dont a fait, et continue à faire, objet ce roman, mais nous voulons, ajouter aux voix savantes et philosophiques qu'elle suscite celle portée par le populaire, le folklorique, de ce roman d'Albert Camus. Albert Camus, écrivain avant tout, Albert Camus, artiste et créateur avant tout. 


\section{Albert Camus, artiste, Albert Camus, créateur}

La Peste d'Albert Camus fait partie du cycle de la Révolte et apparait comme «le premier grand roman français de l'immédiate après-guerre » (Levi-Valensi, 1991: 11). Il vaudra, dix ans après sa parution, le Prix Nobel à son auteur. Ce qui fera de ce roman un grand classique de la littérature française.

6 Unique et universelle à la fois, la critique a longtemps souligné l'aspect philosophique et moraliste de l'œuvre camusienne. Camus le penseur, le journaliste, l'essayiste, le carnétiste chroniquer, le philosophe, le moraliste..., c'est de ces topoïs que partent la majorité des critiques camusiennes pour en faire la partie implicite, mais déterminante, d'un enthymème. Tout alors devient problématisable dans ses textes: ce qui n'est pas consubstantiel au domaine du philosophique ${ }^{4}$ est infléchit à celui de la chronique, mettant de côté ce en tant que quoi Camus se définissait avant tout: Camus, l'artiste, comme il le souligne lui-même lors de sa rencontre avec le poète et dramaturge belge Jean Mogin :

Jean Mogin: On ne sait plus trop souvent, je crois, à confondre Albert Camus l'artiste, le moraliste et même et surtout le philosophe. Monsieur Albert Camus, je voudrais vous demander tout d'abord: que pensez-vous de toute cette confusion dont vous êtes souvent victime?

Albert Camus : Eh bien, c'est une confusion inévitable, mais, si tant est que le point de vue d'un artiste sur lui-même soit le bon, je voudrais insister sur le fait que personnellement, je me sens et je me considère d'abord comme un artiste (Entretien avec Jean Mogin, le 13 septembre 1955, transcription réalisée par nousmême. L'entretien est consultable sur le site de l'INA : <URL : http://www.ina.fr/ audio/PHD98047721>).

7 En tant qu'écrivain d'abord, Camus interroge aussi bien le champ général de la production littéraire européenne que sa propre production qu'il positionne par rapport à son contexte littéraire :

Tout l'effort de l'art occidental est de proposer des types à l'imagination. Et l'histoire de la littérature européenne ne semble pas être autre chose qu'une suite de variations sur ces types et ces thèmes donnés. L'amour racinien est une variation sur un type d'amour qui n'a peut-être pas cours dans la vie. (Camus, $1962: 168$ )

Le classicisme correspond, pour Camus, à la domination des passions individuelles. Même si Camus emprunte au régime d'écriture littéraire dit classique la structure de la tragédie dont il habillera La Peste, il préférera, dans ce roman, une « tentation d'une mise en forme d'une passion collective " (Camus, 1964:140), à une mise en scène d'une expérience individuelle. Un roman qui inscrit sa forme donc dans la tradition classiciste, mais qui s'en éloigne tout aussi bien. Cette double posture sera prise en charge par une orchestration de deux styles: l'un concerne "les actions individuelles» et l'autre concerne, au contraire, «la tragédie collective» (Entretien de Jean Mogin, idem). Un amalgame qui fera de cette épopée des pestiférés une agora où se jouera polyphonie non seulement linguistique, mais également et surtout culturelle, et c'est à cet aspect que nous nous intéressons ici.

9 Nous avons, dans cette perspective, dégagé trois systèmes de croyances, ou de créance, selon la terminologie ethnocritique relative au fléau de la peste. 


\section{Régime de créances positivistes}

La drôle de Peste ? (...) Un des thèmes -possibles- lutte de la médecine et de la religion : les puissances du relatif (et quel relatif !) contre celles de l'absolu. C'est le relatif qui triomphe ou plus exactement qui ne perd pas. (Camus, 1964 : 57)

10 Ce passage avant-textuel place le texte de La Peste sous le signe de l'éternel combat "science vs croyance». Plus intéressant encore pour une approche ethnocritique du texte, il augure une belligérance dans le système axiologique supportée par différents régimes de croyances que le fléau de la peste suscite et est hiérarchisé, dans l'imaginaire du texte, en degrés de « créances $»^{5}$.

11 Nous avons nommé le premier régime : « régime de créance positiviste ", pour désigner cette mise en ordre, cette explication du phénomène épidémique par le prisme d'une cosmologie scientifique. Cette créance semble être portée par le docteur Rieux, et soutenue par toutes les initiatives préfectorales prises dans le but de mettre un terme au fléau.

12 Le docteur Rieux, premier personnage à entrer dans la scène romanesque, est également le premier à être confronté à l'épidémie. C'est lui qui butta, contre le premier rat mort (de la peste) du roman. Phénomène qu'il prend avec insouciance. Il n'y vit aucun mauvais présage, contrairement à la grande majorité de la population oranaise et, à titre d'exemple, le veilleur de nuit que rencontre Tarrou : « A l'hôtel, le veilleur de nuit, qui est un homme de foi, m'a dit qu'il s'attendait à un malheur avec tous ces rats. 'Quand les rats quittent le navire...' « (Camus, $1947: 31$ ).

13 Le médecin, disons-le vite, agit en pur scientifique. Il ne se prononce pas sur le nom de la maladie même après avoir été confronté à plusieurs cas : «- Docteur, disait-elle, qu'est-ce que c'est ? - ça peut être n'importe quoi. Mais il n'y a encore rien de sûr. Jusqu'à ce soir, diète et dépuratif. Qu'il boive beaucoup» (Camus, 1947: 26). Il procède, pour diagnostiquer les pestiférés, devenus de plus en plus nombreux, pragmatiquement. Il appelle un confrère et enquête dans les régions avoisinantes : « il appela encore quelques médecins. L'enquête ainsi menée lui donna une vingtaine de cas semblables en quelques jours » (Camus, $1947: 35$ ).

14 En discutant avec Tarrou à propos du prêche du père Paneloux, Rieux avoue ne pas être croyant. Il ne croit pas en des forces vengeresses qui seraient à l'origine du Fléau. Il choisit plutôt de le combattre par le savoir, plutôt que par la prière :

Peste. Tous luttent- et chacun à sa façon. La seule lâcheté est de se mettre à genoux. On vit sortir des tas de nouveaux moralistes et leur conclusion étaient toujours la même : il faut se mettre à genoux. Mais Rieux répondait : il faut lutter de telle et telle façon (Camus, $1964: 87$ )

15 En faisant de ce personnage central «l'ennemi de Dieu», Camus inscrit dans ce texte l'interminable lutte entre science et religion: "Peste. Rieux dit qu'il était l'ennemi de Dieu puisqu'il luttait contre la mort et que c'était même son métier que d'être l'ennemi de Dieu » (Camus, 1964 : 104).

16 Le médecin, pour revenir à ce personnage central du roman, agit et gère l'épidémie scientifiquement. Cela peut sembler tautologique. Cela, paraitra moins évident quand on entend se dialogiser, dans ce roman, une autre voix que celle de la science, et quand on ne cesse d'y rencontrer, depuis l'incipit jusqu'à l'excipit, des «signes » qui "augurent » jusqu'à surdéterminer l'économie du texte, et « là où les uns voyaient l'abstraction, 
d'autres voyaient la vérité » (Camus, $1947: 89$ ). Ce qui nous offre, dans ce roman, un autre régime de croyance quant à la peste, et nous amène au second point de notre réflexion.

\section{Régime de créances religieuses}

17 Et voilà qu'un autre type de lutte contre ce fléau avait pris forme: les autorités ecclésiastiques avaient décidé de lutter contre la peste par leurs propres moyens, et une semaine de prières avait été organisée. Semaine qui s'est achevée avec le prêche du père Paneloux. Un prêche dont on retrouve l'avant texte biblique dans ce passage carnétiste où Albert Camus retient des passages de l'Ancien et du Nouveau Testament qui lui serviront de fond de toile pour les propos du père :

Peste. Deuxième version.

Bible: Deutéronome, XXVIII, 21; XXXII, 24. Lévitique, XXVI, 25. Amos, IV, 10.

Exode, IX, 4 ; IX, 15 ; XII, 29. Jérémie, XXIV, 10 ; XIV, 12 ; VI, 19 ; XXI, 7 et 9. Ezéchiel,

V, 12 ; VI, 12 ; VII, 15 (Camus, $1964: 54$ )

Dans ce discours sermonnant, l'homme de l'église affirmait ouvertement aux citoyens qu'ils étaient « dans le malheur » car ils l'avaient «mérité » (Camus, 1947 : 91); et le père de dire sur un ton déclaratif: "Vous avez cru qu'il vous suffirait de visiter Dieu le dimanche pour être libres de vos journées. Vous avez pensé que quelques génuflexions le paieraient bien assez de votre insouciance criminelle » (Camus, $1947: 93$ ).

L'hypothèse du fléau comme châtiment divin est ainsi clairement audible dans le prêche du père Paneloux, « jésuite érudit et militant» (Camus, 1947 : 23) qui n'a jamais cru à une simple épidémie. Le sourire de ses yeux avait trahi sa première réaction sur le fléau: " Oh! dit le père, ce doit être une épidémie, et ses yeux sourirent derrière les lunettes rondes » (Camus, 1947 : 23). Il tente d'inscrire, par ce prêche, la peste dans un système de pensée purement monothéiste. Mais quelques éléments discursifs de son énoncé trahissent une autre conception de ce fléau. Nous lisons :

Si aujourd'hui, la peste vous regarde, c'est que le moment de réfléchir est venu. Les justes ne peuvent craindre cela, mais les méchants ont raison de trembler. Dans l'immense grange de l'univers, le fléau implacable battra le blé humain jusqu'à ce que la paille soit séparée du grain. Il y aura plus de paille que de grain, plus d'appelés que d'élus, et ce malheur n'a pas été voulu par Dieu (...). Et battus sur l'aire sanglante de la douleur, vous serez rejetés avec la paille (Camus, 1947 : 92 et passim)

"Immense grange », «blé », " paille», "grain » inscrivent le fléau dans une isotopie tissée par des images paysannes de la peste. La description que donne le narrateur du père Paneloux, au moment du prêche, renforce nos propos : «Il était de taille moyenne, mais trapu, quand il s'appuya sur le rebord de la chaire, serrant le bois entre ses grosses mains, on ne vit de lui qu'une forme épaisse et noire surmontées de deux taches de ses joues, rubicondes sous les lunettes d'acier " (Camus, $1947:$ 91). Description qui semble éloigner le prêcheur de la figure de l'érudit pour le rapprocher de celle du vilain paysan.

Par ailleurs, nous notons que ce prêche respecte la structure de l'exemplum médiéval dans la mesure où il annonce un thème (celui de la peste) : « Ce fut la suite du discours qui fit seulement comprendre à nos concitoyens que, par un procédé oratoire habile, le père avait donné en une seule fois, comme on assène un coup, le thème de son prêche " (Camus, 1947 : 91), intègre subtilement un prothème, autrement dit, une pièce liminaire qui invite l'auditoire à réfléchir (ici celle la peste de l'Egypte antique) : 
Paneloux, tout de suite après cette phrase, en effet, cita le texte de l'Exode relatif à la peste en Egypte et dit: 'La première fois que ce fléau apparait dans l'histoire, c'est pour frapper les ennemis de Dieu. Pharaon s'oppose aux desseins éternels et la peste le fait alors tomber à genoux. (Camus, $1947: 91$ )

Et en tire la Teneur du sermon en commentant la scène intégrée dans son prêche : « $\mathrm{Si}$, aujourd'hui la peste vous regarde, c'est que le moment de réfléchir est venu. Les justes ne peuvent craindre cela, mais les méchants ont raison de trembler » (Camus, 1947 : 92).

L'exemplum est défini par Jean-Claude Schmitt dans Prêche d'exemples. Récits de prédicateurs du Moyen Age (Ed Stock/Moyen Age, Paris, 1985) comme un « récit bref donné comme véridique et destiné à être inséré dans un discours (en général un sermon) pour convaincre un auditoire par une leçon salutaire» (Schmitt, 1985 : 08). Il fut, au Moyen Age, récupéré de tradition antique, jugé comme hérétique, par les clercs pour tenter de mieux se faire entendre du peuple, et ce en adaptant à leur projet d'anciens discours ou modèles discursifs qui lui étaient familiers.

Métaphores, figures paysannes et modèle discursif folklorique nous semblent pénétrer le discours ecclésiastique savant pour y rendre audible une autre voix, un autre régime de croyance qui relève du populaire, et du folklorique. Ce qui nous amène au troisième et dernier point de notre analyse.

\section{Régime de créances folkloriques}

Revenons un instant sur ce passage du prêche du père Paneloux :

Et un bon ange apparut visiblement, qui donnait des ordres au mauvais ange qui portait un épieu de chasse et il lui ordonnait de frapper les maisons; et autant de fois qu'une maison recevait de coups, autant y avait-il de morts qui en sortaient (...) Mes Frères, dit-il avec force, c'est la même chasse mortelle qui court aujourd'hui dans nos rues. (Camus, $1947: 93$ )

«Épieu de chasse » et «chasse mortelle » inscrivent ce passage dans une isotopie de la chasse. La peste est ainsi perçue comme un fléau qui poursuit et "chasse», au sens propre du terme, la population oranaise. La peste, introduit par un élément du sauvage, les rats, se transforme ici en "chasse sauvage ", relevant d'une croyance européenne folklorique médiévale.

Le mythe de la chasse sauvage renvoie, selon les études réunies par Philippe Walter dans Le Mythe de la chasse sauvage dans l'Europe médiévale (éditions Honoré Champion, Paris, 1997), à une croyance aux origines préchrétiennes qui expliquent certains phénomènes météorologiques (Tempêtes, vent violent...) par le passage d'une horde de chasseurs (morts) en fureur (parce que punis pour avoir transgressé une loi sacrée, religieuse le plus souvent); horde bruyante accompagnée d'animaux, condamnée à errer et à poursuivre éternellement un gibier qui les aurait détournés de la messe du dimanche. Le sauvage, le recommencement perpétuel, la horde sauvage, mais initialement humaine, bruit et désordre, sont donc les thèmes essentiels qui se dégagent de ce scénario mythique.

Dans ce roman camusien, c'est le sauvage qui introduit le fléau: les rats, bêtes qui viennent d'un espace qui se situe en dehors de l'univers organisé des hommes, le perturbent. Le discours du concierge à propos du premier rat trouvé dans son immeuble illustre parfaitement cette séparation établie entre le domestique et le sauvage, et le désordre occasionné par l'intrusion du dernier dans le premier : 
La présence de ce rat mort lui avait paru seulement bizarre tandis que, pour le concierge, elle constituait un scandale. La position de ce dernier était d'ailleurs catégorique : il n'y avait pas de rats dans la maison. Le docteur eut beau l'assurer qu'il y en avait un sur le palier du premier étage, et probablement mort, la conviction de M. Michel restait entière. Il n'y avait pas de rats dans la maison, il fallait donc qu'on eut apporté celui-ci du dehors. (Camus, $1947: 15$ ). concierge, lui, est scandalisé: il ne peut y avoir de rats dans l'espace domestique (affirmation réitérée deux fois de suite). On les a apportés du "dehors », d'un espace séparé du domestique.

L'apparition du sauvage dans le domestique va jusqu'à "ensauvager » la plupart des protagonistes : C'est le cas de toute la famille qu'on rencontre au restaurant de l'hôtel :

- On ne parle pas de rats à table, Philippe. Je vous interdis à l'avenir de prononcer ce mot.

- Votre père a raison, a dit la souris noire.

Les deux caniches ont piqué le nez dans leurs pâtes et la chouette a remercié d'un signe de tête qui n'en disait pas long. (Camus, 1947 : 32).

C'est également le cas de Grand qui « avait l'allure du sanglier » (Camus, 1947 : 55), du passeur qu'avait rencontré Rambert en vue de son évasion : "Le cheval encensa une fois de plus et Rambert approuva sans passion (...)» (Camus, 1947: 138), de même pour Tarrou : «Dans la pénombre du palier, Tarrou avait l'air d'un grand ours vêtu de gris » (Camus, 1947 : 117).

32 Le fléau introduit par cet élément du sauvage prend possession de la ville, l'enferme et réduit ses habitants à un perpétuel recommencement :

Pendant des semaines, nous fûmes réduits alors à recommencer sans cesse la même lettre, à recopier les mêmes appels, si bien, qu'au bout d'un certain temps, les mots qui d'abord étaient sortis tout saignants de notre cœur se vidaient de leur sens (...) En d'autres circonstances, d'ailleurs, nos concitoyens auraient trouvé une issue dans une vie plus extérieure et plus active. Mais, en même temps, la peste les laissait oisifs, réduits à tourner en rond dans leur ville morne et livrés, jour après jour, aux jeux décevants du souvenir. Car, dans leurs promenades sans but, ils étaient amenés à passer toujours par les mêmes chemins, et, la plupart du temps, dans une si petite ville, ces chemins étaient précisément ceux qu'à une autre époque ils avaient parcourus avec l'absent (...) Et par là, échoués à mi-distance de ces abîmes et de ces sommets, ils flottaient plutôt qu'ils ne vivaient, abandonnés à des jours sans direction et à des souvenirs stériles, ombres errantes qui n'auraient pu prendre force qu'en acceptant de s'enraciner dans la terre de leur douleur. (Camus, $1947: 67 ; 69 ; 70$ et 72 )

Dans l'intrigue de La Peste, les personnages principaux sont décrits dès les premières pages, même si certains d'entre eux, à l'image du journaliste Rambert ne reviennent que plus tard dans le roman pour accomplir leur parcours narratif. Il s'agit, d'un groupe d'hommes (les femmes ne sont qu'évoquées) que la narration regroupe par la sonnette d'alarme que tire la peste, comme des chasseurs ameutés à la vue d'un gibier.

Tout comme la meute de la Chasse sauvage, la population d'Oran s'était détournée de Dieu, et le prêche du père Paneloux ne manque pas de mettre l'accent sur ce point. D'ailleurs, dès l'ouverture du roman, la description de la population d'Oran annonce une cité dont les habitants ne réservent aucune place à la pratique d'un quelconque culte : Ils «travaillent beaucoup, mais toujours pour s'enrichir. Ils s'intéressent surtout au commerce et ils s'occupent d'abord, selon leur expression, de faire des affaires » (Camus, 
1947 : 12). Les adverbes «surtout » et « d'abord» expriment la primauté de la principale occupation des habitants: celle du commerce, et de l'enrichissement. Les plaisirs des «joies simples» sont laissés pour les samedis et dimanches : «Le dimanche matin, par exemple, les bains de mer font une concurrence sérieuse à la messe » (Camus, $1947: 90$ ), ce qui est, rappelons-le encore, la principale cause du châtiment de la Mesnie Hellequin.

Mais ceci n'est pas le seul « signe prémonitoire du roman » : la nature revêtit dès l'incipit, une fonction augurale :

Les curieux événements qui font le sujet de cette chronique se sont produits en 194., à Oran. De l'avis général, ils n'y étaient pas à leur place, sortant un peu de l'ordinaire. A première vue, Oran est, en effet, une ville ordinaire et rien de plus qu'une préfecture française de la côte algérienne. La cité elle-même, on doit l'avouer, est laide. D'aspect tranquille, il faut quelque temps pour apercevoir ce qui la rend différente de tant d'autres villes commerçantes, sous toutes les latitudes. Comment faire imaginer, par exemple, une ville sans pigeons, sans arbres, et sans jardins, où l'on ne rencontre ni battements d'ailes ni froissement de feuilles, un lieu neutre pour tout dire? Le changement des saisons ne s'y lit que dans le ciel. Le printemps s'annonce seulement par la qualité de l'air ou par les corbeilles de fleurs que des petits vendeurs remmènent des banlieues; c'est un printemps qu'on vend sur les marchés. Pendant l'été, le soleil incendie les maisons trop sèches et couvre les murs d'une cendre grise ; on ne peut plus vivre alors que dans l'ombre des volets clos. En automne, c'est, au contraire, un déluge de boue. Les beaux jours viennent seulement en hiver. (Camus, $1947: 11$ )

La description de la ville fait ici, selon notre hypothèse de lecture, figure de signe prémonitoire. L'adjectif «curieux » sur lequel s'ouvre la narration du roman jette sur l'intrigue un voile de mystère. Le lecteur sait, de prime abord, qu'il s'agira d'une histoire étrange, d'une histoire qui sort de l'ordinaire, alors que la peste en tant qu'épidémie est un thème universel. La ville est ainsi annoncée comme « différente ». Dénudée de faune et de flore, elle subit les caprices du temps climatique: le printemps n'y a plus rien de printanier, l'été et l'automne y sont des théâtres apocalyptiques. Seul l'hiver est symbole de beau-temps. Curieux climat pour une ville méditerranéenne.

Le temps climatique accompagne également le parcours narratif du fléau, et en annonce le moindre changement :

Le lendemain, 30 avril, une brise déjà tiède soufflait dans un ciel bleu et humide. Elle apportait une odeur de fleur qui venait des banlieues les plus lointaines. Les bruits du matin dans les rues semblaient plus vifs, plus joyeux que l'ordinaire. Dans toute notre petite ville, débarrassée de la sourde appréhension où elle avait vécu pendant la semaine, ce jour là était celui du renouveau. (Camus, 1947 : 27).

Ces signes fonctionnent, dans la logique culturelle du roman, comme des intersignes, c'est-à-dire des mantiques narratives qui annoncent, et déterminent les événements qui défileront au fils de la narration ${ }^{6}$.

Le temps climatique finit par s'associer à la peste pour "chasser » les citoyens d'Oran : « le soleil poursuivait nos concitoyens dans tous les coins de rue et, s'ils s'arrêtaient, il les frappait alors » (Camus, $1947: 106)$.

40 Les habitants de la ville d'Oran, telle la horde de la chasse sauvage, semblent être condamnés dans un mouvement perpétuel, dans une mise en abîme de la chasse : ils chassent alors qu'ils sont eux même chassés. Ils poursuivent et chassent l'équilibre, le quotidien qu'ils avaient perdu avec l'apparition du fléau qui les chasse lui-même à cause de ce quotidien négligeant qui leur attirât un châtiment divin. Condamnés, même une fois le siège de la peste levé, à vivre dans la peur d'un éventuel retour. 
41 configuration de croyances composite. Un mot qui se charge, au fil de la narration, de valeurs culturelles plus ou moins hétérogènes, et contamine, de son hybridité, les personnages du roman. Rieux par exemple, personnage central du roman, et qui se révèle à la fin en être le narrateur, porte tour à tour les voix des croyances dont fait objet le fléau. société, combien même algéro-française, mais algérienne avant tout; moment où toutes les mutations fondamentales s'y jouaient, révèle une autre dimension d'un roman qui écrit ses propres croyances, sa propre culture. Le fléau ne cesse d'être problématisé, ouvrant la voie à une circulation fluide entre des modes de conception habituellement conflictuels, tissant un fil symbolique de concession entre divers systèmes de croyance (scientifique, savant, populaire...) et révélant une polyphonie culturelle qui articule le discours romanesque à d'autres discours (scientifique, religieux : Camus révèle lui-même les sources de sa documentation: historique, médicale, religieuse) qui ne sont pas les siens. Une sorte de pensée sauvage, primaire et non primitive, qui bricole les rapports entre entités hétérogènes, révélant la pensée humaniste et conciliatrice de Camus, quand à la question de l'Algérie. Une pensée qui se concrétise bien plus tard dans son appel « pour la trêve civile en Algérie », en 1956.

\section{BIBLIOGRAPHIE}

BAKHtine, Mikhaïl (1970). La Poétique de Dostoievski. Paris : Seuil.

BAKHTINE, Mikhaïl (1978). Esthétique et théorie du roman. Paris : Gallimard, « Bibliothèque des idées"

CAMUS, Albert (1947). La Peste. Paris : Gallimard, « Folio ».

CAMUS Albert (1962). Carnet I, Mai 1935- Février 1942, Paris : Gallimard, [consulté le 10/10/2013]

$<U R L$ : http://classiques.uqac.ca/classiques/camus_albert/carnets_I/camus_carnets_t1.pdf>, <DOI : http://dx.doi.org/doi:10.1522/030160477>.

CAMUS, Albert (1964). Carnet II. Janvier 1942 - mars 1951. Paris : Gallimard, [consulté le 10/10/2013] <URL : http://classiques.uqac.ca/classiques/camus_albert/carnets_II/camus_carnets_t2.pdf>, <DOI : http://dx.doi.org/doi:10.1522/030150886>.

CNOCKAERT, Véronique, PRIVAT, Jean-Marie, SCARPA, Marie (dir.) (2011). L'Ethnocritique de la littérature. Québec: Presses Universitaires du Québec, « Approches de L'imaginaire ».

Dufournet, Jean (dir.) (1997). Le Mythe de la Chasse sauvage dans l'Europe médiévale. Paris : Honoré Champion.

HELL, Bertrand (1994). Le Sang noir. Chasse et mythe du Sauvage en Europe. Paris : Champs

Flammarion.

LeCouteuX, Claude (1999). Chasses fantastiques et cohortes de la nuit au Moyen Age. Paris : IMAGO. 
LeVI-VALENSI, Jacqueline (1991). La Peste d'Albert Camus. Paris : Gallimard, « Folio ».

MENARD, Sophie (Hiver 2014, à paraitre). « Les Créances du roman : le revenant dans Thérèse Raquin de Zola », @nalyses. Revue de critique et de théorie littéraire, pp. 330-358, [consulté le 10/10/2013]

<URL :https://uottawa.scholarsportal.info/ojs/index.php/revue-analyses/article /view/ 972/832 $>$.

PRIVAT, Jean-Marie (1994). Bovary Charivari, Essai d'ethnocritique. Paris : Centre National de la Recherche Scientifique.

PRIVAT, Jean-Marie et SCARPA, Marie (dir.) (2010). Horizons ethnocritiques. Nancy : Presses

Universitaires de Nancy, « ethnocritiques».

Schмiтt, Jean-Claude (1985). Prêcher d'exemples. Récits de prédicateurs du Moyen Age. Paris : Stock/

Moyen Age, « Moyen Age ».

Entretien avec Jean Mogin, le 13 septembre 1955, consultable sur le site de l'INA :

<URL : http://www.ina.fr/audio/PHD98047721>.

\section{NOTES}

1. Albert Camus l'avoue dans l'entretien sus-cité.

2. Notamment Carnets I et Carnets II, dans la mesure où le projet de l'écriture de La Peste y est souvent interrogé.

3. Nous prenons comme base théorique de notre réflexion l'approche ethnocritique qui se définit essentiellement comme «l'étude de la pluralité et de la variation culturelles constitutives des œuvres littéraires telles qu'elles peuvent se manifester dans la configuration d'univers discursifs plus ou moins hétérogènes » (Cnockaert, Privat, Scarpa, $2011: 02$ ).

4. Même l'explication (thématique et/ou structurelle) des textes camusiens y est fortement rattachée.

5. Le système de créance désigne, dans la taxinomie ethnocritique, « un outil de mise en ordre logique, et comme un système d'interprétation des signes, des faits, des destins ». Les travaux ethnocritiques ( $c f$. www.ethnocritique.com pour une vue panoramique des travaux de la discipline) préfèrent cette dénomination à celle de croyance dans la mesure où « elle ne suppose pas, contrairement à la 'croyance', une axiologie fondée sur le sacré ni liée à des formes magicoreligieuses. Un groupe peut avoir un système de créance, auquel cas ce système fait partie intégrante de sa cosmologie, comprise dans un sens anthropologique comme un 'système du monde d'un groupe social'« (Ménard, $2014: 330$ ).

6. $C f$. les travaux de Jean-Marie Privat, notamment le dernier chapitre de Bovary Charivari, Pour cette question $d^{\prime}$ « intersignes ».

\section{RÉSUMÉS}

Dans cet article, nous proposons d'étudier La Peste d'Albert Camus dans une perspective qui croise poétique des textes littéraires et ethnologie du symbolique. Dans cette optique, nous avons 
voulu concentrer notre réflexion sur les différents systèmes de croyances/créances qui gravitent autour du fléau de la peste afin de dégager la polyphonie culturelle constitutive de cette œuvre.

In this paper, we propose to study La Peste by Albert Camus in a perspective that crosses poetic literary texts and symbolic anthropology. In this context, we wanted to focus our thinking on the different systems of beliefs/créances that revolve around the scourge of the plague in order to identify the constitutive cultural polyphony of this work.

INDEX

Keywords : Peste (La), Camus (Albert), ethno criticism, cultural polyphony, regime of créances

Mots-clés : Peste (La), Camus (Albert), ethnocritique, polyphonie culturelle, régime de créances

\section{AUTEUR}

\section{BENKHODJA AMMAR}

CREM, Un. de Lorraine

a.benkhodja[at]gmail.com 